\title{
APORTE DE HOJARASCA DEL MANGLE NEGRO (Avicennia germinans L.) EN EL CARIBE COLOMBIANO
}

\author{
Álvaro Javier Martínez-Ballesteros ${ }^{1}$ \\ Carlos Mario Plaza-Rodríguez ${ }^{1}$ \\ Giovany Guevara ${ }^{2}$
}

Recibido el 22 de julio de 2013 y aprobado el 12 de octubre de 2013

\section{RESUMEN}

A pesar que los manglares son reconocidos a nivel mundial como ecosistemas altamente productivos y ecológicamente importantes, en la actualidad, su extensión se ha reducido significativamente debido a las actividades humanas en las zonas costeras. Por lo tanto, es necesario investigar su dinámica y respuestas bajo el creciente y sostenido impacto del cambio climático y antropogénico. Durante dos estaciones climáticas contrastantes (sequía, lluvia) se estudió la producción de hojarasca del mangle negro Avicennia germinans L. en la bahía El Uno cerca al municipio de Turbo, noroccidente de Colombia $\left(8^{\circ} \mathrm{N}\right)$. El aporte de materia orgánica fue separado por estructuras (hojas, ramas, flores y frutos) donde se registró un aporte significativamente mayor de hojas. La biomasa seca total durante ambas estaciones fue de 7,09 $\pm 9,66$ y 5,36 $\pm 5,67 \mathrm{~g}$ $\mathrm{m}^{-2} \mathrm{dí}^{-1}$. Los valores mínimos y máximos fueron de 0,63-8 y 0,49$3,65 \mathrm{~g} \mathrm{~m}^{-2}$ día ${ }^{-1}$ durante la estación seca y lluviosa, respectivamente. Se detectaron diferencias significativas entre las estructuras orgánicas pero no entre las estaciones climáticas analizadas. Se discuten algunos impactos naturales y antropogénicos pasados y actuales, que afectan la dinámica de los manglares, al igual que la importancia de la producción de hojarasca para la productividad primaria y secundaria, en zonas altamente perturbadas del Caribe colombiano.

\section{PALABRAS CLAVE}

Golfo de Urabá, Mar Caribe, manglares, materia orgánica costera, productividad.

\section{CONTRIBUTION OF BLACK MANGROVE (Avicennia germinans L.) FALLEN LEAVES IN THE COLOMBIAN CARIBBEAN AREA}

\section{ABSTRACT}

Although mangroves are recognized worldwide as highly productive and ecologically important ecosystems, at present its extent has been significantly reduced due to human activities in coastal areas. Therefore, it is necessary to investigate their dynamics and responses under the growing and sustained climatic and anthropogenic impacts. During two contrasting seasons (drought, rain) the fallen leaves production of the black mangrove Avicennia germinans L. was investigated at El Uno Bay, near Turbo Municipality, North-Western Colombia $\left(8^{\circ} \mathrm{N}\right)$. Organic matter input was sorted by structure (leaves, branches, flowers, fruits) with 
leaves registering the highest significant values. Total dry biomass during both seasons was $7.09 \pm 9.66$ and $5.36 \pm 5.67 \mathrm{~g} \mathrm{~m}^{-2}$ day ${ }^{-1}$. The lowest and highest organic matter values were 0.63-8 and 0.49-3.65 $\mathrm{g} \mathrm{m}^{-2}$ day $^{-1}$ during the dry and rainy season, respectively. Significant differences between organic structures but not between seasons analyzed were detected. Some relevant past and present natural and anthropogenic impacts affecting the mangroves dynamics are discussed, as well as the importance of fallen leaves input to the primary and secondary production in highly disturbed zones of the Colombian Caribbean area.

\section{KEY WORDS}

Gulf of Urabá, Caribbean Sea, coastal organic matter, mangroves, productivity.

\section{INTRODUCCIÓN}

Los manglares son ecosistemas estratégicos que crecen en zonas tropicales y subtropicales costeras (entre $35^{\circ} \mathrm{N}-38^{\circ} \mathrm{S}$ ) y presentan, generalmente, altas tasas de productividad primaria (Hogarth, 2007; Sánchez-Carrillo et al., 2009). A su vez, son ecosistemas que contribuyen con el reciclaje de nutrientes en ambientes estuarinos y costeros, a través del aporte de materia orgánica y los procesos de descomposición asociados (López-Portillo \& Ezcurra, 1985; Lee, 1995; Hogarth, 2007; Sánchez-Andrés et al., 2010). Estos ecosistemas suministran una gran variedad de bienes (e.g., madera como combustible, para construcción y pesca, medicamentos, y alimento) y servicios (e.g., protección costera, control de erosión e inundaciones, apoyo a pesquerías, entre otros) (Primavera, 1998; Tam \& Wong, 2002; Sanjurjo \& Welsh, 2005). Sin embargo, actualmente existe una fuerte presión antropogénica sobre ellos, y a la vez, están sometidos al efecto del cambio climático (YáñezArancibia, Twilley \& Lara, 1998; Gilman et al., 2008).

La hojarasca (que incluye toda la materia orgánica vegetal que se desprende de los árboles), al igual que el crecimiento subsuperficial de las raíces, constituyen una fuente significativa de carbono (C) orgánico hacia el sedimento de los ecosistemas de manglar, ya que estos tienen la capacidad de atrapar eficientemente el material en suspensión dentro de la columna de agua (Alongi et al., 2005). Asimismo, el aporte de hojarasca en estos ambientes puede representar hasta un tercio de la productividad primaria neta. No obstante, existen otras fuentes que también suministran una entrada importante de $\mathrm{C}$ orgánico, entre las que se citan: material alóctono ribereño o marino, producción autóctona por micro o macroalgas bénticas o epifíticas y producción local por el fitoplancton dentro de la columna de agua (Lee, 1999; Kristensen et al., 2008; SánchezAndrés et al., 2010; Kamruzzaman et al., 2012). Como consecuencia, los ecosistemas de manglar son sitios de procesamiento intenso de nutrientes con un impacto potencialmente alto sobre el balance global del carbono (Lee, 1999; Mfilinge, Atta \& Tsuchiya, 2002; Kristensen et al., 2008). La dinámica de la producción y descomposición de hojarasca en los manglares, 
presenta una variación geográfica y estacional (López-Portillo \& Ezcurra, 1985; Tam et al., 1998; Sánchez-Andrés et al., 2010), al tiempo que depende de los rasgos fenológicos de las especies; donde el crecimiento y senescencia de tejidos y órganos, pueden estar directamente relacionados con factores bióticos y abióticos (Sharma et al., 2012).

A nivel mundial existen numerosos estudios sobre la producción y descomposición de hojarasca en manglares, especialmente en las regiones tropicales de Australia, Estados Unidos, Tailandia y Malasia (véase Tam et al., 1998; Tam \& Wong, 2002; Komiyama, Ong \& Poungparn, 2008; Kristensen et al., 2008; Feller et al., 2010). La mayor parte de la información publicada está relacionada con los géneros Rhizophora y Avicennia (Tam et al., 1998). En Colombia, las investigaciones sobre productividad de manglares han sido realizadas por Álvarez (2003), Galeano et al. (2010) y CortésCastillo \& Rangel-Ch. (2011). Asimismo, se reportan estudios en la zona del Caribe colombiano, particularmente relacionados con el impacto antropogénico (Blanco-Libreros, 2009; Arroyave-Rincón, Blanco \& Taborda, 2012; Blanco \& Castaño, 2012; Blanco et al., 2012). En el costado occidental del golfo de Urabá, Cadavid (2010) realizó la evaluación del aporte, descomposición y química de la hojarasca de Rhizophora mangle L. En la laguna costera El Uno, no se reportan trabajos relacionados con el aporte y dinámica de la hojarasca de manglar; sin embargo, se han realizado otras investigaciones con invertebrados, peces y productividad (ver referencias en Amortegui-Torres, Taborda-Marín \& Blanco, 2013).

Aunque los manglares han sido resaltados como sistemas heterotróficos (Kristensen et al., 2008), la importancia del aporte de hojarasca para mantener su metabolismo, es aún desconocida, particularmente en zonas con altas tasas de impacto antropogénico como el golfo de Urabá (Blanco et al., 2012). El objetivo del presente estudio fue evaluar el aporte de la hojarasca del mangle negro, Avicennia germinans L. (Jiménez \& Lugo, s.f.) en la laguna costera El Uno (golfo de Urabá), como una manera de detectar su potencial influencia en la dinámica de nutrientes en zonas costeras del Caribe colombiano y contribuir con un potencial manejo sustentable, donde se requiere de mayor conocimiento en el ciclo de nutrientes de estos ecosistemas estratégicos.

\section{MATERIALES Y MÉTODOS}

El golfo de Urabá es el sector más alejado del Mar Caribe, en este se presenta una transición entre playas arenosas, el delta estuarino del río Atrato (entre otros ríos de menor caudal) y costas rocosas, las cuales limitan al occidente con la República de Panamá. Su longitud es de alrededor de $80 \mathrm{~km}$ y presenta un ancho variable entre 5 y 50 km (García-Valencia, 2007). Se extiende desde Punta Arenas al norte de la costa oriental hasta Cabo Tiburón en la costa occidental (Chevillot et al., 1993).

La laguna costera El Uno, se localiza en el delta del río Turbo ( $8^{\circ} 05^{\prime}$ $\mathrm{N}-76^{\circ} 44^{\prime} \mathrm{O}$ ), en la parte central de la costa oriental del golfo de 
Urabá (Caribe colombiano) (Figura 1). Tiene una extensión aproximada de $1,2 \mathrm{~km}^{2}$ y una profundidad promedio de $1 \mathrm{~m}$ (García \& Correa, 2006). En la zona noroccidental de la laguna, se encuentra un manglar conformado desde la línea de costa hacia la zona continental por las especies $R$. mangle, A. germinans y Laguncularia racemosa (L.) Gaertn. f., respectivamente (CORPOURABÁ, 2003; Correa \& Palacio, 2008). Observaciones in situ del manglar, indican que se encuentra bajo una alta intervención antropogénica (Figura 2).

En la zona se presenta un periodo de lluvias abundante durante abril a noviembre y lluvias escasas de enero a marzo. La precipitación promedio anual fluctúa entre 2900 a 4100 mm, la temperatura ambiente oscila entre 26 a $28^{\circ} \mathrm{C}$, la humedad relativa del aire registra promedios mensuales entre 83 y $86 \%$, el brillo solar presenta un valor promedio de 5 horas diarias, los vientos predominantes son los Alisios del Norte, el régimen de marea es semidiurno con una amplitud inferior a 0,5 m. También se registran cambios en la salinidad del agua, asociados estos a las condiciones climáticas, presentándose valores menores en la época de baja precipitación, debido a que los vientos Alisios retienen los aportes superficiales de agua dulce provenientes del río Atrato en la bahía Colombia (Chevillot et al., 1993).
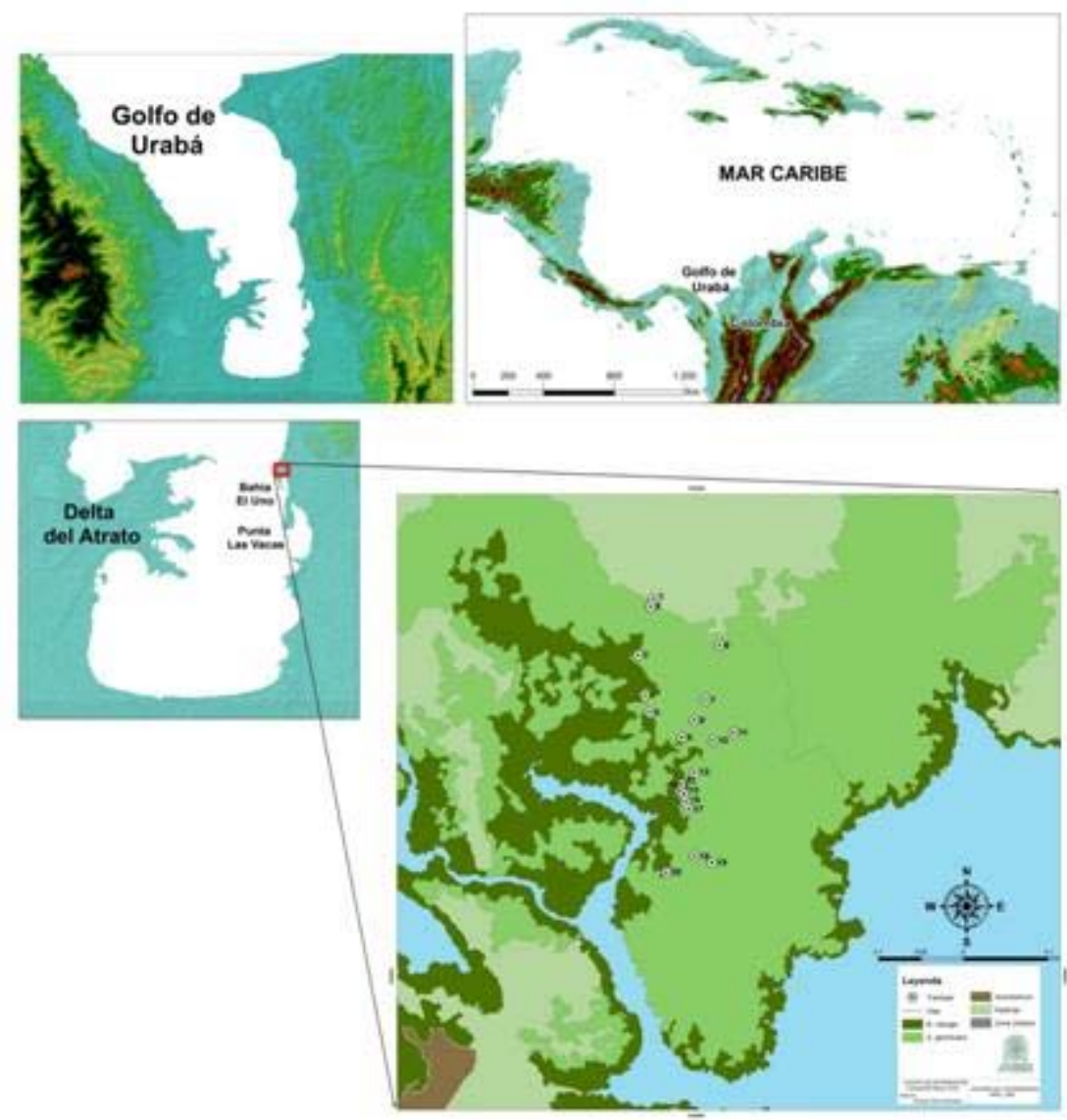

Figura 1. Ares de estudio: bshís El Uno, golfo de Urabá, noroccidente de Colombis. Se indice la ubicación de las trampas en la vegetación circundante de la laguns costera E Uno (derecha-sbajo). 


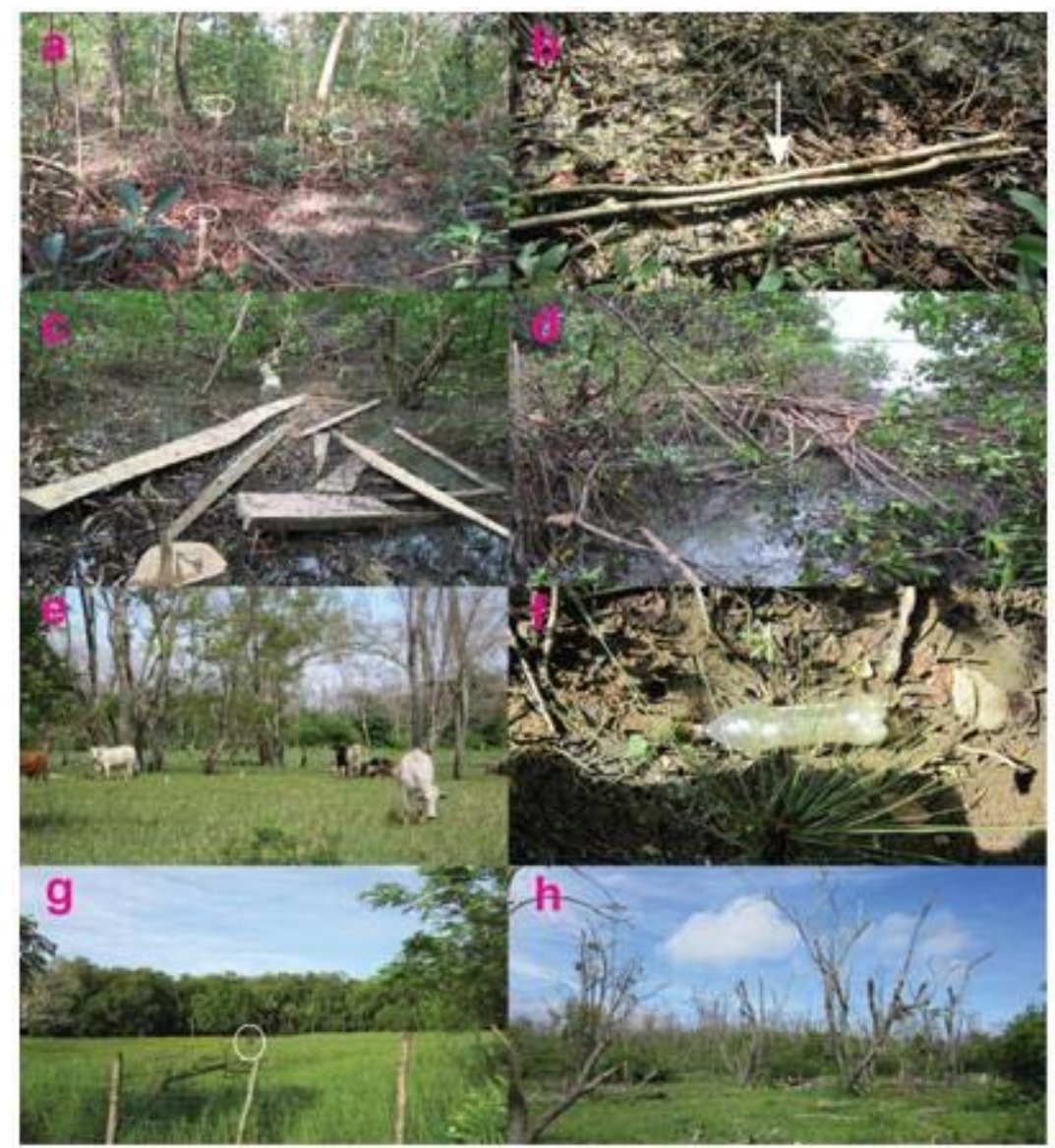

Futn: Los suas:s

Figura 2. Algunos de los impsctos antropogénicos y naturales observados en cercaniss a áres de estudio. Se muestran áress de corts "selectiva" en el corredor de mangle (a), al osrecer con propositos de extracción de madera para construcción de viviends. postes/cercas (b, c, d), impactos porganadería (e), lugar de almacenamiento temporal de bssuras [flujo de mares (f)], extracción de leña por pobladores de la zons (g) y mortalidad de los mengles producids al parecer por efectos de sedimentsción (h), como consecuencis de le inundsción provocsds por el río Turbo (Pérez, 2012).

\section{Aporte de hojarasca, determinación del peso seco y comparación entre épocas climáticas}

El aporte de hojarasca fue evaluado durante dos épocas climáticas contrastantes: lluvia (noviembre-diciembre de 2012) y sequía (enero-febrero de 2013). En una zona del ecosistema de manglar entre la laguna El Uno y el delta del río Turbo, se ubicó una sección de $120 \mathrm{~m}$ paralela a la línea de costa, donde se instalaron 20 trampas de hojarasca $(50 \times 50 \times 50 \mathrm{~cm}$, ojo de malla de $5 \mathrm{~mm})$ por cada época de muestreo. Estas se localizaron a 2,5 $\mathrm{m}$ de altura de la base de los árboles de $A$. germinans y se sujetaron con una cuerda desde los extremos (Figura 3). Debido a diversas restricciones logísticas, la recolección de las muestras se realizó a los 45 días de instaladas las trampas, durante cada época climática. El material orgánico de cada trampa fue colocado en bolsas de papel y transportado al laboratorio de la Universidad de Antioquia seccional Urabá, para su fraccionamiento (hojas, ramas, flores y frutos), secado y determinación del peso seco (g). El secado del material orgánico se realizó en un horno convencional a $80^{\circ} \mathrm{C}$ durante $48 \mathrm{~h}$ y posteriormente se pesó en una balanza semi- 
analítica (OHAUS ${ }^{\circledR}$ Pioneer ${ }^{\mathrm{TM}}$, 0,1 mg de precisión). Para detectar diferencias significativas del peso seco entre las estructuras orgánicas y las épocas de muestreo, se realizó un análisis de varianza de dos vías (variables: estructura orgánica, época de muestreo), después de la verificación de los supuestos de normalidad y homocedasticidad, y considerando las trampas como réplicas por cada época. La comparación entre las medias de los diferentes niveles del factor significativo, se realizó con la prueba a posteriori de Tukey. Los datos de peso seco $\left(\mathrm{g} \mathrm{m}^{-2}\right.$ día $\left.{ }^{-1}\right)$ fueron convertidos para estimar la producción anual de hojarasca para el área de estudio. Los datos fueron analizados con el software STATISTICA versión 10 (StatSoft, 2011).

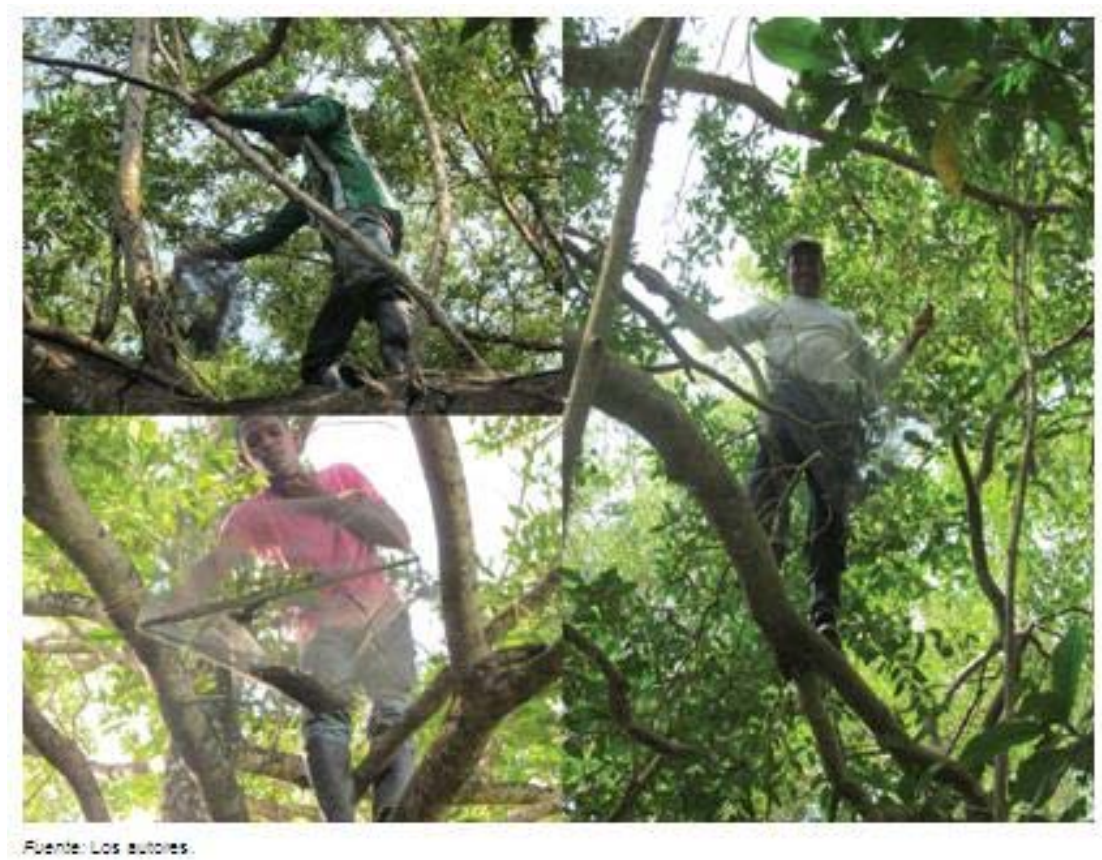

Figura 3. Ubicación de las trampas de hojersscs utilizadss pars is colects del materis orgénico durante el periodo de estudio.

\section{RESULTADOS}

\section{Producción hojarasca y separación por estructuras orgánicas}

Los valores de producción total de hojarasca (promedio \pm desviación

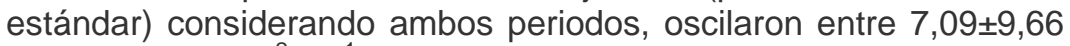
y 5,36 $5,67 \mathrm{~g} \mathrm{~m}^{-2} \mathrm{día}^{-1}$. Los valores mínimos y máximos del aporte de materia orgánica fueron de 0,63-8 y 0,49-3,65 $\mathrm{g} \mathrm{m}^{-2}$ día ${ }^{-1}$ durante el periodo seco y lluvioso, respectivamente. Durante el periodo seco, el aporte promedio de hojarasca fue de 2,52 $\pm 1,75 \mathrm{~g} \mathrm{~m}^{-2} \mathrm{dí}^{-1}$, mientras que en el periodo lluvioso fue de $1,91 \pm 0,79 \mathrm{~g} \mathrm{~m}^{-2} \mathrm{dí}^{-1}$. Al considerar la producción de hojarasca por estructuras durante los dos periodos, los valores fueron: hojas $(12,22 \pm 5,07)>$ ramas $(5,78 \pm 7,93)>$ flores $(5,01 \pm 10,3)>$ frutos $\left(1,89 \pm 2,11 \mathrm{~g} \mathrm{~m}^{-2}\right.$ día $\left.{ }^{-1}\right)$, y fue mayor para la época seca (Figura 4). Se detectaron diferencias significativas en la producción de las diferentes estructuras orgánicas pero no entre los periodos climáticos evaluados (Tabla 1). 
El aporte total de hojarasca estimado para el área de estudio fue de 8 ton ha ${ }^{-1}$ año ${ }^{-1}$.

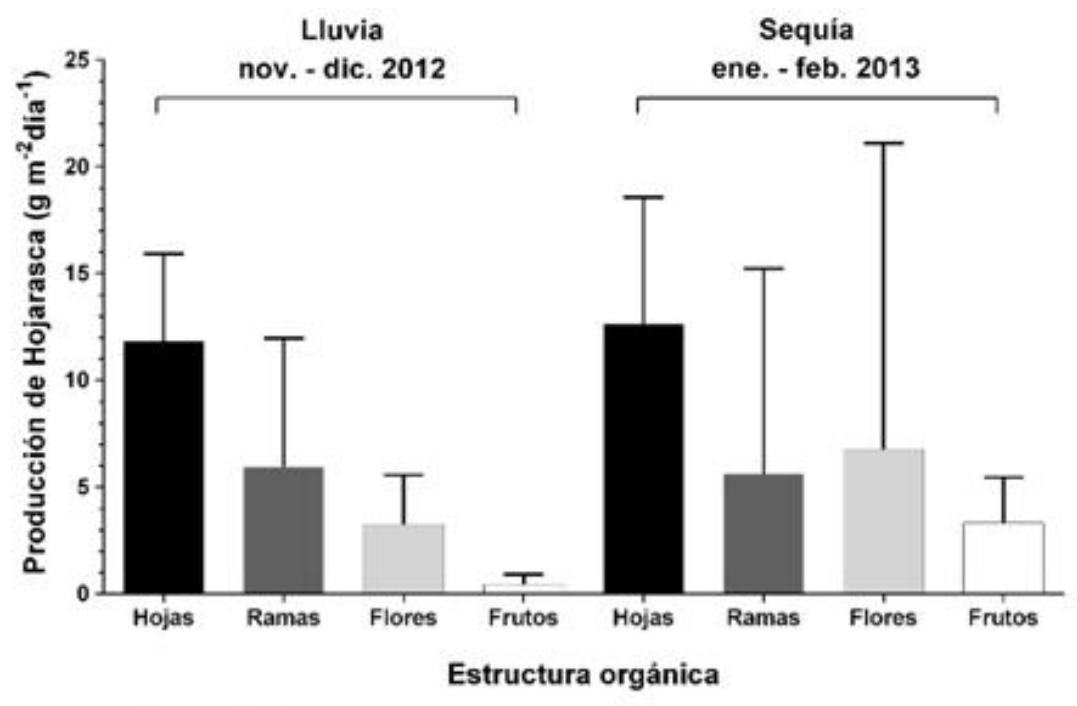

Fuente: Esta investigacion

Figura 4. Aporte de hojarasca de Avicennia germinans en dos épocas contrastantes, en la laguna costera EI Uno, golfo de Urabá, Caribe colombiano. Se muestran los valores promedio y la desviación estándar.

Tabla 1. Análisis de varianza de dos vías para detectar diferencias significativas entre las estructuras orgánicas de Avicennia germinans y entre épocas de colecta.

\begin{tabular}{lcccccc}
\hline \multicolumn{1}{c}{ Factor } & SC & GL & MC & F & P & $\begin{array}{c}\text { Prueba de } \\
\text { Tukey }\end{array}$ \\
\hline Estructura orgánica & 2255.663 & 3 & 751.888 & 15.243 & $<0,001$ & $\mathrm{H}=(\mathrm{R}=\mathrm{F} \mid=\mathrm{Fr})$ \\
Periodo(lluvia/sequia) & 119.232 & 1 & 119.232 & 2.417 & 0,122 & \\
Error & 7645.239 & 155 & 49.324 & & & \\
\hline
\end{tabular}

H: Hojas. H: Kamas. Hl: rlores. tr: trutos

Fuente: Esta investigacion.

\section{DISCUSIÓN}

A pesar de los avances en el estudio de los manglares en Colombia, aún persisten zonas con escasas investigaciones como el caso del golfo de Urabá (Blanco et al., 2011). Los manglares del golfo presentan un desarrollo estructural, comparativamente mayor que los de otras áreas del Caribe colombiano (Sánchez-Páez et al., 1997), con una dominancia de Rhizophora mangle y representación escasa de $A$. germinans (Blanco et al., 2011). Sin embargo, en el área seleccionada para el estudio, existe una mayor abundancia de A. germinans (Hoyos, Urrego \& Lema, 2011; Pérez, 2012) a pesar del creciente y sostenido impacto antropogénico. Estudios recientes han indicado que los manglares de las bahías de Turbo y El Uno (que representan un 2,9\% de los bosques de manglar del golfo de Urabá), las cuales están separadas del cuerpo principal del golfo por grandes barreras depositadas por el río Turbo, presentan bosques altamente intervenidos por el hombre debido a cambios de uso del suelo, tala, ganadería, sobrepesca, entre otros, cerca de la zona urbana del municipio de Turbo, el más grande en las riberas del 
golfo (Blanco et al., 2012; Ortiz \& Blanco, 2012; Amortegui-Torres et al., 2013; Gómez \& Bernal, 2013). No obstante, existen acciones estratégicas promovidas por la autoridad ambiental (CORPOURABÁ, com. pers.) para la regeneración de las especies de mangle y manejo sustentable del mismo, que permiten reducir el impacto de la tala y extracción de madera del ecosistema de manglar.

El aporte de hojarasca en los ecosistemas de manglar, ha sido ampliamente utilizado como una medida de la productividad primaria y un elemento fundamental en la productividad secundaria, como sustrato para consumidores y descomponedores; al igual que su papel en la formación del suelo, el ciclo de nutrientes y las transformaciones biogeoquímicas (Lugo \& Snedaker, 1974; Lee, 1999; López-Portillo \& Ezcurra 2002; Hogarth, 2007; CoronadoMolina et al., 2012). Los manglares son plantas esclerófilas siempreverdes con aportes de materia orgánica a lo largo del ciclo anual; sin embargo, tienden frecuentemente a concentrar la caída de hojarasca en estaciones determinadas (López-Portillo \& Ezcurra, 2002). Nuestros resultados mostraron un aporte mayor de hojarasca durante la temporada seca, con una dominancia de las hojas sobre otras estructuras orgánicas. Este comportamiento fue similar al reportado por Arreola-Lizárraga, Flores-Verdugo \& Ortega-Rubio (2004) en el golfo de California, México. Sin embargo, comparativamente estos autores registraron menores valores promedio de aporte diario de hojarasca $\left(0,48 \mathrm{~g} \mathrm{~m}^{-2} \mathrm{dí}^{-1}\right)$. Asimismo, Aké-Castillo, Vázquez \& López-Portillo (2006) reportaron que la producción anual de hojarasca, donde las hojas representaron la fracción orgánica mayor (70\%), siguió un patrón estacional con dos eventos máximos en la época seca y uno durante la lluviosa. Por el contrario, Coronado-Molina et al. (2012) registraron un efecto estacional y significativamente mayor durante la estación lluviosa, aunque los valores de producción de hojarasca fueron contrastantes entre períodos, en relación con nuestro estudio (lluvia $=2,4$; sequía $\left.=1,8 \mathrm{~g} \mathrm{~m}^{-2} \mathrm{dí}^{-1}\right)$. No obstante, aunque parece ocurrir una relación positiva entre la precipitación (estacionalidad) y el aporte de hojarasca, cada componente estructural sigue una secuencia diferente (López-Portillo \& Ezcurra, 1985, 2002; Aké-Castillo et al., 2006). Algunos autores han indicado que el mayor aporte de hojarasca está directamente relacionado con la salinidad y esta a su vez con la precipitación (Yáñez-Arancibia et al., 1998; Feller et al., 2010). Los bajos niveles de salinidad son factores que contribuyen significativamente al aumento de la productividad (Hernández \& Mullen, 1978; López et al., 1988; Ochoa, 1988). En la bahía El Uno, estos niveles son más bajos en épocas secas y mayores en periodos húmedos (Correa \& Palacio, 2008). Esto se debe a que los vientos Alisios del noreste, atrapan en la bahía Colombia los aportes de agua dulce del río Atrato (Chevillot et al., 1993; Correa \& Palacio, 2008). Los bajos niveles de salinidad que presenta la bahía El Uno, se pueden asociar con los mayores aportes de hojarasca registrados durante la época seca. Sin embargo, nuestros resultados deben ser considerados como preliminares dada la duración del periodo muestreado por cada época climática y a la ausencia de potenciales correlaciones que no pudieron ser analizadas, tales como aspectos de composición y estructura del bosque y la influencia de variables ambientales (e.g., precipitación, evaporación, radiación, temperatura y salinidad; López-Portillo \& 
Ezcurra, 2002; Cortés-Castillo \& Rangel-Ch., 2011; CoronadoMolina et al., 2012). Por lo tanto, se recomienda que para un estudio detallado sobre la dinámica de la hojarasca de los bosques de manglar en la bahía El Uno, se realicen estudios del aporte durante al menos un año e incluir otros aspectos relacionados con la composición y estructura de los bosques de mangle, así como el registro de otras variables ambientales, que puedan influir sobre la dinámica de nutrientes en estos ecosistemas costeros.

\section{CONSIDERACIONES FINALES}

Es necesario continuar con programas de monitoreo -idealmente bajo una perspectiva de largo plazo- de los ecosistemas de manglar en la zona estudiada y en los demás ambientes costeros de Colombia. Se requiere además de la valoración de la producción primaria y secundaria asociada con estos ecosistemas. Asimismo, es necesario lograr la capacitación de propietarios y habitantes de las zonas de manglar para promover un manejo sustentable y mantener un suministro constante de bienes y servicios para la población y la fauna; al igual que, establecer un área mínima de corredor para evitar la ampliación de la frontera ganadera y garantizar la conservación de la estructura y función del manglar. Finalmente, se deben apoyar los planes y programas de recuperación sugeridos por la autoridad ambiental, la academia y los pobladores rurales y urbanos del golfo de Urabá.

\section{AGRADECIMIENTOS}

A los profesores Jenny Leal, Tatiana Correa y Jairo Zapata por el préstamo de la balanza para el registro del peso seco de las estructuras orgánicas, a Genis Gomes por facilitar el horno para el secado del material, a la Universidad de Antioquia a través de la Corporación Académica Ambiental por el apoyo brindado a la presente investigación, y a la Universidad de Caldas por las facilidades dadas a G. Guevara para su participación en este proyecto. Agradecemos las sugerencias del comité editorial y de los revisores anónimos, que mejoraron significativamente la versión original del manuscrito.

\section{REFERENCIAS}

- Aké-Castillo, J. A., Vázquez, G. y López-Portillo, J. (2006). Litterfall and Decomposition of Rhizophora mangle L. in a Coastal Lagoon in the Southern Gulf of Mexico. Hydrobiologia, 559(1), 101-111.

- Alongi, D. M., Pfitzner, J., Trott, L. A., Tirendi, F., Dixon, P. y Klumpp, D. W. (2005). Rapid sediment accumulation and microbial mineralization in forests of the mangrove Kandelia candel in the Jiulongjiang Estuary, China. Estuarine, Coastal and Shelf Science, 63(4), 605-618. 
- Álvarez León, R. (2003). Los manglares de Colombia y la recuperación de sus áreas degradadas: revisión bibliográfica y nuevas experiencias. Madera y Bosques, 9(1), 3-25.

- Amortegui-Torres, V., Taborda-Marín, A. y Blanco, J. F. (2013). Edge effect on a Neritina virginea (Neritimorpha, Neritinidae) population in a black mangrove stand (Magnoliopsida, Avicenniaceae: Avicennia germinans) in the Southern Caribbean. Pan-American Journal of Aquatic Sciences, 8(2), 68-78.

- Arreola-Lizárraga, J. A., Flores-Verdugo, F. J. y Ortega-Rubio, A. (2004). Structure and litterfall of an arid mangrove stand on the Gulf of California, Mexico. Aquatic Botany, 79(2), 137-143.

- Arroyave-Rincón, A., Blanco, J. F. y Taborda, A. (2012). Exportación de sedimentos desde cuencas hidrográficas de la Vertiente Oriental del Golfo de Urabá: Influencias climáticas y antrópicas. Revista Ingenierías Universidad de Medellín, 11(20), 13-30.

- Blanco-Libreros, J. F. (2009). Banana Crop Expansion and Increased River-borne Sediment Exports to the Gulf of Urabá, Caribbean Coast of Colombia. AMBIO: A Journal of the Human Environment, 38(3), 181-183.

- Blanco, J. F., Londoño-Mesa, M., Quan-Young, L., Urrego-Giraldo, L., Polanía, J., Osorio, A., Bernal, G. y Correa, I. (2011). The Urabá Gulf Mangrove Expedition of Colombia. ISME/GLOMIS, 9(3), 8-10.

- Blanco, J. F. y Castaño, M. C. (2012). Efecto de la conversión del manglar a potrero sobre la densidad y tallas de dos gasterópodos en el delta del río Turbo (Golfo de Urabá, Caribe Colombiano). Revista de Biología Tropical, 60(4), 1707-1719.

- Blanco, J. F., Estrada, E. A., Ortiz, L. F. y Urrego, L. E. (2012). Ecosystem-Wide Impacts of Deforestation in Mangroves: The Urabá Gulf (Colombian Caribbean) Case Study. ISRN Ecology, 2012, 1-14.

- Cadavid González, S. (2010). Caída, descomposición y química de la hojarasca de Rhizophora mangle a lo largo de un gradiente hidrodinámico en la bahía Marirrío, Golfo de Urabá. Universidad de Antioquia, Medellín.

- Chevillot, P., Molina M., A., Giraldo, L. y Molina Márquez, C. (1993). Estudio geológico e hidrológico del Golfo de Urabá. Boletín Científico del Centro de Investigaciones Oceanográficas e Hidrográficas, 14, 79-90.

- Coronado-Molina, C., Álvarez-Guillén, H., Day, J. W. J., Reyes, E., Pérez, B. C., Vera-Herrera, F. y Twilley, R. (2012). Litterfall dynamics in carbonate and deltaic mangrove ecosystems in the Gulf of Mexico. Wetlands Ecology and Management, 20(2), 123-136.

- CORPOURABÁ. (2003). Caracterización y Zonificación de los Manglares del Golfo de Urabá, Departamento de Antioquia.

- Correa Rendón, J. D. y Palacio Baena, J. A. (2008). Ictiofauna de las áreas de manglar en las bahías Turbo y El Uno, Golfo de Urabá. Gestión y Ambiente, 11(3), 43-54.

- Cortés-Castillo, D. V. y Rangel-Ch., J. O. (2011). Los bosques de mangle en un gradiente de salinidad en la Bahía de Cispatá - Boca Tinajones, departamento de Córdoba-Colombia. Caldasia, 33(1), 155-176.

- Feller, I. C., Lovelock, C. E., Berger, U., McKee, K. L., Joye, S. B. y Ball, M. C. (2010). Biocomplexity in Mangrove Ecosystems. Annual Review of Marine Science, 2(1), 395-417.

- Galeano Galeano, E., Mancera Pineda, J. E. y Medina Calderón, J. H. (2010). Efecto del sustrato sobre la descomposición de hojarasca 
en tres especies de mangle en la Reserva de Biosfera Seaflower, Caribe Colombiano. Caldasia, 32(2), 411-424.

- García-Valencia, C. (Ed.). (2007). Atlas del Golfo de Urabá: una mirada al Caribe de Antioquia y Chocó. Santa Marta: Instituto de Investigaciones Marinas y Costeras-INVEMAR y Gobernación de Antioquia.

- García, J. M. y Correa, J. C. (2006). Macroinvertebrados y peces asociados a las raíces sumergidas del mangle rojo (Rhizophora mangle) en las Bahías de Turbo y El Uno, Golfo de Urabá. Universidad de Antioquia, Medellín.

- Gilman, E. L., Ellison, J., Duke, N. C. y Field, C. (2008). Threats to mangroves from climate change and adaptation options: A review. Aquatic Botany, 89(2), 237-250.

- Gómez, E. y Bernal, G. (2013). Influence of the environmental characteristics of mangrove forests on recent benthic foraminifera in the Gulf of Urabá, Colombian Caribbean. Ciencias Marinas, 39(1), 69-82.

- Hernández, J. y Mullen, K. (1978). Productividad primaria en un manglar del Pacífico Colombiano. Tomo I. Paper presented at the Mem. Sem. Oc. Pacífico Sudamericano, Cali (Colombia). Septiembre 1-5 de 1976.

- Hogarth, P. (2007). The Biology of Mangroves and Seagrasses (2nd. ed.). New York: Oxford University Press.

- Hoyos, R., Urrego Giraldo, L. E. y Lema, A. (2011). Respuesta de la regeneración natural de los manglares del delta del Río Turbo y la Bahía El Uno (Golfo de Urabá-Colombia), a la variabilidad ambiental y climática intra-anual. Universidad Nacional de Colombia, Medellín.

- Jiménez, J. A. y Lugo, A. E. (s.f.). Avicennia germinans (L.) L. SOITF-SM-4. New Orleans, LA: U.S. Department of Agriculture, Forest Service, Southern Forest Experiment Station. Recuperado de http://www.fs.fed.us/global/iitf/Avicenniagerminans.pdf

- Kamruzzaman, M., Sharma, S., Rafiqul Hoque, A. T. M. y Hagihara, A. (2012). Litterfall of three subtropical mangrove species in the family Rhizophoraceae. Journal of Oceanography, 68(6), 841-850.

- Komiyama, A., Ong, J. E. y Poungparn, S. (2008). Allometry, biomass, and productivity of mangrove forests: A review. Aquatic Botany, 89(2), 128-137.

- Kristensen, E., Bouillon, S., Dittmar, T. y Marchand, C. (2008). Organic carbon dynamics in mangrove ecosystems: A review. Aquatic Botany, 89(2), 201-219.

- Lee, S. Y. (1995). Mangrove outwelling: a review. Hydrobiologia, 295(1-3), 203-212.

- . (1999). Tropical mangrove ecology: Physical and biotic factors influencing ecosystem structure and function. Australian Journal of Ecology, 24(4), 355-366.

- López-Portillo, J. y Ezcurra, E. (1985). Litter fall of Avicennia germinans L. in a one-year cycle in a mudflat at the Laguna de Mecoacan, Tabasco, Mexico. Biotropica, 17(3), 186-190.

- (2002). Los manglares de México: una revisión. Madera y Bosques, 8(Es1), 27-51.

- López, J. M., Stoner, A. W., García, J. R. y García Muniz, I. (1988). Marine food webs associated with Caribbean island mangrove wetlands. Acta Científica, 2(2-3), 94-123.

- Lugo, A. E. y Snedaker, S. C. (1974). The Ecology of Mangroves. Annual Review of Ecology and Systematics, 5(1), 39-64. 
- Mfilinge, P., Atta, N. y Tsuchiya, M. (2002). Nutrient dynamics and leaf litter decomposition in a subtropical mangrove forest at Oura Bay, Okinawa, Japan. Trees, 16(2-3), 172-180.

- Ochoa Bejarano, G. (1988). Algunas características funcionales del manglar de la Ciénaga Grande de Santa Marta, con énfasis en Rhizophora mangle y Avicennia germinans. Universidad del Valle, Cali.

- Ortiz, L. F. y Blanco, J. F. (2012). Distribución de los gasterópodos del manglar, Neritina virgínea (Neritidae) y Littoraria angulifera (Littorinidae) en la Ecorregión Darién, Caribe colombiano. Revista de Biología Tropical, 60(1), 219-232.

- Pérez, J. E. (2012). Diagnóstico de la extracción de los recursos maderables en los manglares de Turbo en Puntas Las Vacas y Yarumal (Antioquia) y el análisis de la presencia de Acrostichum aureum L. y Montrichardia arborescens L. Universidad de Antioquia, Medellín.

- Primavera, J. H. (1998). Mangroves as Nurseries: Shrimp Populations in Mangrove and Non-mangrove Habitats. Estuarine, Coastal and Shelf Science, 46(3), 457-464.

- Sánchez-Andrés, R., Sánchez-Carrillo, S., Alatorre, L. C., Cirujano, S. y Álvarez-Cobelas, M. (2010). Litterfall dynamics and nutrient decomposition of arid mangroves in the Gulf of California: Their role sustaining ecosystem heterotrophy. Estuarine, Coastal and Shelf Science, 89(3), 191-199.

- Sánchez-Carrillo, S., Sánchez-Andrés, R., Alatorre, L. C., Angeler, D. G., Álvarez-Cobelas, M. y Arreola-Lizárraga, J. A. (2009). Nutrient fluxes in a semi-arid microtidal mangrove wetland in the Gulf of California. Estuarine, Coastal and Shelf Science, 82(4), 654-662.

- Sánchez-Páez, H., Álvarez-León, R., Pinto-Nolla, F., SánchezAlférez, A. S., Pino-Rengifo, J. C., García-Hansen, I. y AcostaPeñaloza, M. T. (1997). Diagnóstico y zonificación preliminar de los manglares del Caribe de Colombia. Bogotá D.C.: Ministerio del Medio Ambiente.

- Sanjurjo Rivera, E. y Welsh Casas, S. (2005). Una descripción del valor de los bienes y servicios ambientales prestados por los manglares. Gaceta Ecológica, 74, 55-68.

- Sharma, S., Kamruzzaman, M., Rafiqul Hoque, A. T. M. y Hagihara, A. (2012). Leaf phenological traits and leaf longevity of three mangrove species (Rhizophoraceae) on Okinawa Island, Japan. Journal of Oceanography, 68(6), 831-840.

- StatSoft, Inc. (2011). STATISTICA (data analysis software system), version 10. www.statsoft.com

- Tam, N. F. Y., Wong, Y. S., Lan, C. Y. y Wang, L. N. (1998). Litter production and decomposition in a subtropical mangrove swamp receiving wastewater. Journal of Experimental Marine Biology and Ecology, 226(1), 1-18.

- Tam, N. F. Y. y Wong, Y. (2002). Conservation and sustainable exploitation of mangroves in Hong Kong. Trees, 16(2-3), 224-229.

- Yáñez-Arancibia, A., Twilley, R. R. y Lara Domínguez, A. L. (1998). Los ecosistemas de manglar frente al cambio climático global. Madera y Bosques, 4(2), 3-19. 
1. Programa de Ecología de Zonas Costeras, Corporación Académica Ambiental, Universidad de Antioquia seccional Urabá, Turbo (Antioquia, Colombia).

2. Departamento de Desarrollo Rural y Recursos Naturales, Facultad de Ciencias Agropecuarias, Universidad de Caldas, Manizales (Caldas, Colombia).

Autor para correspondencia. Giovany Guevara: ggcolombia@gmail.com

Para citar este artículo: Martínez-Ballesteros, Á. J., PlazaRodríguez, C. M. \& Guevara, G. (2014). Aporte de hojarasca del mangle negro (Avicennia germinans I.) en el Caribe colombiano. Revista Luna Azul, 38, 218-230. Recuperado de http://lunazul.ucaldas.edu.co/index.php?option=content\&task=v iew\&id $=903$ 